\title{
The Validity and Reliability of GPS Units for Measuring Distance in Team Sport Specific Running Patterns
}

\author{
Denise Jennings, Stuart Cormack, Aaron J. Coutts, \\ Luke Boyd, and Robert J. Aughey
}

\begin{abstract}
Purpose: To assess the validity and reliability of distance data measured by global positioning system (GPS) units sampling at 1 and $5 \mathrm{~Hz}$ during movement patterns common to team sports. Methods: Twenty elite Australian Football players each wearing two GPS devices (MinimaxX, Catapult, Australia) completed straight line movements $(10,20,40 \mathrm{~m})$ at various speeds (walk, jog, stride, sprint), changes of direction (COD) courses of two different frequencies (gradual and tight), and a team sport running simulation circuit. Position and speed data were collected by the GPS devices at 1 and $5 \mathrm{~Hz}$. Distance validity was assessed using the standard error of the estimate $( \pm 90 \%$ confidence intervals $[\mathrm{CI}])$. Reliability was estimated using typical error (TE) $\pm 90 \%$ CI (expressed as coefficient of variation [CV]). Results: Measurement accuracy decreased as speed of locomotion increased in both straight line and the COD courses. Difference between criterion and GPS measured distance ranged from $9.0 \%$ to $32.4 \%$. A higher sampling rate improved validity regardless of distance and locomotion in the straight line, COD and simulated running circuit trials. The reliability improved as distance traveled increased but decreased as speed increased. Total distance over the simulated running circuit exhibited the lowest variation (CV 3.6\%) while sprinting over $10 \mathrm{~m}$ demonstrated the highest $(\mathrm{CV}$ $77.2 \%$ at $1 \mathrm{~Hz}$ ). Conclusion: Current GPS systems maybe limited for assessment of short, high speed straight line running and efforts involving change of direction. An increased sample rate improves validity and reliability of GPS devices.
\end{abstract}

Keywords: time-motion analysis, accuracy, precision, acceleration.

Quantifying the movement demands of team sport players during training and matches provides valuable information that can be used to better understand the

Jennings is with the Institute of Sport, Exercise and Active Living, School of Sport and Exercise Science, Victoria University, Melbourne, Australia, and the High Performance Unit, Essendon Football Club, Melbourne, Australia. Cormack is with the High Performance Unit, Essendon Football Club, Melbourne, Australia. Coutts is with the High Performance Unit, Essendon Football Club, Melbourne, Australia, and the School of Leisure, Sport \& Tourism, University of Technology, Sydney, Australia. Boyd is with the Institute of Sport, Exercise and Active Living, School of Sport and Exercise Science, Victoria University, Melbourne, Australia, and the Western Bulldogs Football Club, Melbourne, Australia. Aughey is with the Institute of Sport, Exercise and Active Living, School of Sport and Exercise Science, Victoria University, Melbourne, Australia, and the Western Bulldogs Football Club, Melbourne, Australia. 
demands of competition and develop specific training programs. Until recently, measurement and quantification of player movement demands has been difficult as it has required manual analysis with only one player being analyzed at a time. ${ }^{1}$ However, with recent technological developments there are now many systems that can analyze movement patterns of many players in team sports simultaneously, including manual and computer-assisted video-based time motion analysis systems, triangulation of miniature electronic transmitters and global positioning system (GPS) devices. ${ }^{2,3}$ Specifically, GPS technology is used by many sports to quantify movement demands during training and competition. ${ }^{4-8}$

The use of video-based time-motion analysis (TMA) has been well documented ${ }^{2,9-11}$ however, more recently there is a growing body of literature examining the use of GPS for game and player analysis. ${ }^{4,5,12,13}$ Indeed, GPS technology has the potential to provide a more comprehensive, accurate and automated examination of player movements in invasion style team sports (eg, hockey, rugby union, rugby league, soccer and Australian Football). However, few studies have investigated the reliability and validity of GPS technology for the assessment of team sport movement patterns. ${ }^{5-8,13}$ Additionally, most studies have assessed the validity and reliability of these GPS devices with distance and positional data recorded at 1 $\mathrm{Hz},{ }^{5-8}$ with relatively few utilizing $5 \mathrm{~Hz}$ data. ${ }^{13}$

Recent developments in technology now allow position data to be collected at $5 \mathrm{~Hz}$ during match play and training. This increase in sample rate may enhance the validity and reliability of GPS for team sport applications. To date, however, only one study has examined the validity and reliability of $5 \mathrm{~Hz}$ GPS devices, assessing cricket-specific movement patterns. ${ }^{13}$ The results demonstrated that $5 \mathrm{~Hz}$ devices have an acceptable validity and reliability for estimating longer distances (600-8800 m) in walking and striding activities (ie, $\mathrm{CV}<3 \%$ ), but were poor (ie, $\mathrm{CV}>15 \%$ ) for shorter sprints of between 20 to $40 \mathrm{~m} .{ }^{13}$ The efficacy of these devices for assessing movement demands specific to team sports such as the football codes is not well understood.

Critical movements to performance in team sports are the ability to maximally accelerate, decelerate and change direction at speed over a short distance. ${ }^{3}$ For example, Australian football (AF), soccer, rugby union and hockey players' exhibit similar sprint distances, typically between 10 to $20 \mathrm{~m} .^{8,12,14,15}$ Several studies have shown that increased sprint and repeated-sprint performance, ${ }^{14-19}$ particularly with the ball,,$^{10}$ are important for team performance. In addition, the ability to change direction is important in these sports. ${ }^{14,20,21}$ Dawson et al ${ }^{19}$ investigated players' movement patterns in AF and demonstrated that more than half the number of sprints performed in a match involved at least one change of direction. It is therefore necessary to be able to accurately measure these characteristics in match play. More recently, a $5 \mathrm{~Hz}$ sample rate has been introduced in an attempt to provide greater accuracy for assessing these brief high-intensity efforts.

Therefore, the aims of this study were to assess the reliability and validity at both $1 \mathrm{~Hz}$ and $5 \mathrm{~Hz}$ of the MinimaxX GPS device for the assessment of different movement patterns and distances common to team sport play.

\section{Methods}

Twenty elite Australian football players (mean $\pm \mathrm{SD}$ ) age, stature and body mass: $24 \pm 4 \mathrm{y}, 188 \pm 7 \mathrm{~cm}$, and $87 \pm 9 \mathrm{~kg}$ respectively participated in the study. This 
study conformed to the National Health and Medical Research Council's Statement on Human Experimentation. All athletes gave informed consent following full disclosure of procedures.

\section{Experimental Design}

This study evaluated the validity and reliability of distance data collected from GPS units at 1 and $5 \mathrm{~Hz}$ during movement patterns common to team sports. Specifically, the accuracy of these devices was assessed during straight line running at various speeds, during two change of direction (COD) courses, and during a team sport running circuit. This research design allowed for the assessment of team-sport specific movement patterns of increasing intensities, complexity and specificity. During each trial subjects wore two GPS units (MinimaxX, Team 2.5, Catapult Innovations, Scoresby, Australia) in a custom made harness with the units located on the right and left scapula, approximately $25 \mathrm{~cm}$ apart. The antennas of each unit were exposed to allow a clear satellite reception. Trials were completed on a grassed AF field, clear of large buildings to enhance satellite reception. The mean \pm SD number of satellites during data collection for the straight line, COD and simulated team sport running circuit were $8.2 \pm 0.6,8.3 \pm 0.4$ and $8.2 \pm 0.6$, respectively. The horizontal dilution of position (HDOP) is a reflection of the geometrical arrangement of satellites and is related to both the accuracy and quality of the signal. Values can range between 1 and a maximum value of $50,{ }^{22}$ with an ideal HDOP of 1 indicating that one satellite is directly overhead with the remainder equally spaced around the horizon. Higher values indicating that the position fix is entirely unreliable. ${ }^{23}$ The mean HDOP during data collection was $1.5 \pm 0.28,1.26$ \pm 0.09 and $1.25 \pm 0.06$ for straight line, COD and simulated team sport running circuit, respectively. To assess the influence of sample rate, data for each trial was collected and analyzed at 1 and $5 \mathrm{~Hz}$.

Electronic timing gates (Smart Speed, Fusion Sport, Queensland, Australia) were used to obtain a criterion sprint time for a known distance (accurate to $0.01 \mathrm{~s}$ ). The GPS estimated distance for the time taken on each course as measured by the timing gates was determined with Logan Plus v4.2.3 software (Catapult Innovations, Scoresby, Australia) for each trial. The start time for each trial was determined by the first increase above zero on the velocity trace. ${ }^{13}$ The time from timing lights was used to indicate the end of the movement for each known distance.

\section{Straight-Line Running}

Subjects commenced from a stationary position and completed two trials of each self selected speed of walking, jogging, striding and sprinting. Timing gates set up at the $0,10,20,40 \mathrm{~m}$ points measured subject time over these distances.

\section{Change of Direction}

Subjects walked, jogged, strode and sprinted through two different courses (Figure 1) of known distance $(40 \mathrm{~m})$ and angle of COD $\left(90^{\circ}\right)$. Players commenced from a stationary position and followed a painted course marked on the ground. At each COD, poles were used to ensure players tracked the correct pathway as close to the known distance as possible. 


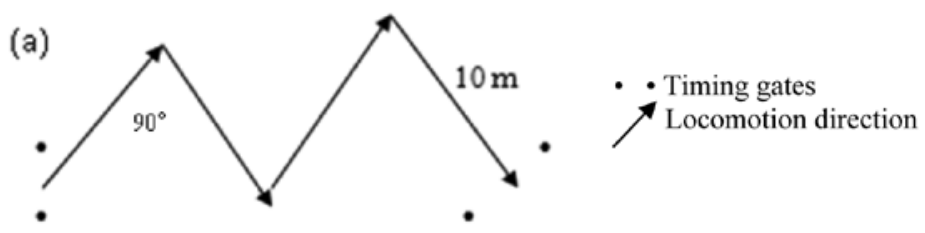

(b)

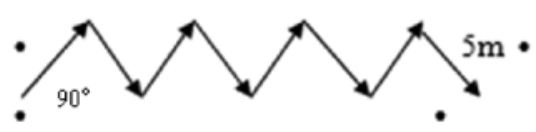

Figure 1 - Change of direction (COD) course: (a) Gradual $10 \mathrm{~m}$ COD. $4 \times 10 \mathrm{~m}$ straights with $3 \times 90^{\circ} \mathrm{COD}(\mathrm{b})$ Tight $5 \mathrm{~m}$ COD. $8 \times 5 \mathrm{~m}$ straights with $7 \times 90^{\circ} \mathrm{COD}$.

\section{Simulated Team Sport Running Circuit}

A sample of players $(n=10)$ completed five trials of a marked team sport running circuit $\left(140 \mathrm{~m}\right.$ ) modified from Bishop et $\mathrm{al}^{24}$ (Figure 2). The circuit included, two maximal sprints, a zigzag COD, three periods of walking, three periods of jogging, one striding effort and a deceleration to a complete stop. Measurement of the circuit

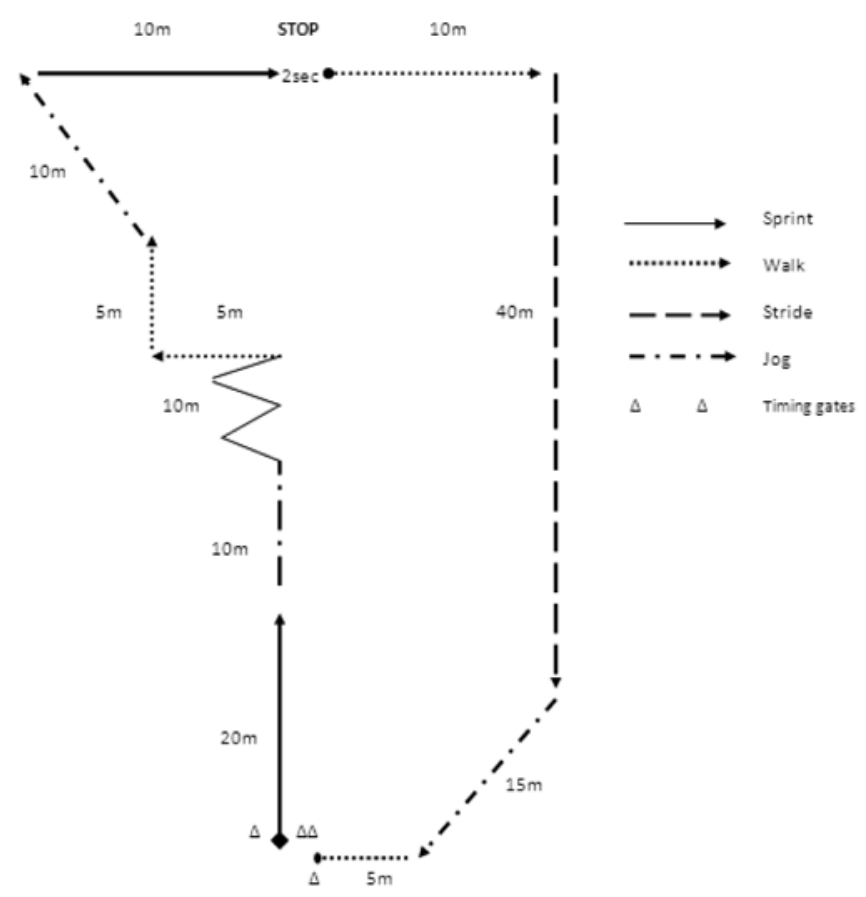

Figure 2 - Modified team sport circuit. ${ }^{24}$ 
was made with a calibrated measuring tape and goniometer for COD sections. The participants were instructed to start from a stationary position and follow a marked course on the grassed playing field, completing each circuit within one minute. At the COD section, poles and painted lines were used to ensure players tracked the correct pathway as close to the known distance as possible. Each player performed a familiarization trial before the beginning of the test period and was verbally instructed as to which motion was required for each section of the circuit.

\section{Statistical Analyses}

Validity was assessed using the standard error of the estimate (SEE). The SEE was calculated as the standard deviation $( \pm 90 \% \mathrm{CI})$ of the $\%$ difference between the known distance and the GPS recorded distance for each trial at both $1 \mathrm{~Hz}$ and $5 \mathrm{~Hz}$. The percentage difference $( \pm 90 \% \mathrm{CI})$ between the reference distance and GPS distance was also calculated to indicate bias. ${ }^{13}$

The reliability of measures in each locomotor pattern for the $40 \mathrm{~m}$ straight line, COD and simulated team sport running circuit sampled at both $1 \mathrm{~Hz}$ and $5 \mathrm{~Hz}$ was estimated using the typical error (TE) $\pm 90 \%$ CI expressed in absolute and as a percentage (coefficient of variation [CV]). The smallest worthwhile change (SWC), defined as the smallest change of practical importance was calculated as $0.2 \times$ the between subject standard deviation. ${ }^{25}$ As a result, variables were considered capable of detecting the SWC if the TE was less than or equal to SWC ${ }^{25}$ Once TE was established, it was divided into criterion categories, being rated as good $(\mathrm{CV}<5 \%)$, moderate $(\mathrm{CV}$ $5 \%$ to $10 \%)$ or poor $(\mathrm{CV}>10 \%)$, based on previous recommendations. ${ }^{26}$

\section{Results}

\section{Validity}

The accuracy of the GPS, for measuring distance during straight line movements at different speeds, at 1 and $5 \mathrm{~Hz}$ is presented in Table 1 . As the speed of locomotion increased over a given distance, validity decreased. Larger errors were associated with jogging, striding and sprinting over $10 \mathrm{~m}$ (Table 1). The validity improved as the distance increased for all locomotion speeds at both 1 and $5 \mathrm{~Hz}$ (Table 1). With the exception of walking at $1 \mathrm{~Hz}$ in the tight course, validity decreased as the locomotion speed increased in both the gradual and tight COD (Table 2). In addition, the higher sample rate improved the measurement accuracy of both the gradual and tight COD trials resulting in error less than $11.7 \%$.

The validity of the total distance during the simulated team sport running circuit at 1 and $5 \mathrm{~Hz}$ was $3.6 \pm 0.6 \%$ and $3.8 \pm 0.6 \%$ respectively.

\section{Bias}

The GPS substantially underestimated the criterion distance when striding and sprinting over short distances $(10$ and $20 \mathrm{~m}$ ) at both 1 and $5 \mathrm{~Hz}$ (Table 3). As the distance increased, the magnitude of the error decreased when jogging, striding and sprinting (Table 3). GPS underestimated the criterion distance during the tight COD trial at all locomotion speeds (Table 4). A higher sampling rate decreased the 


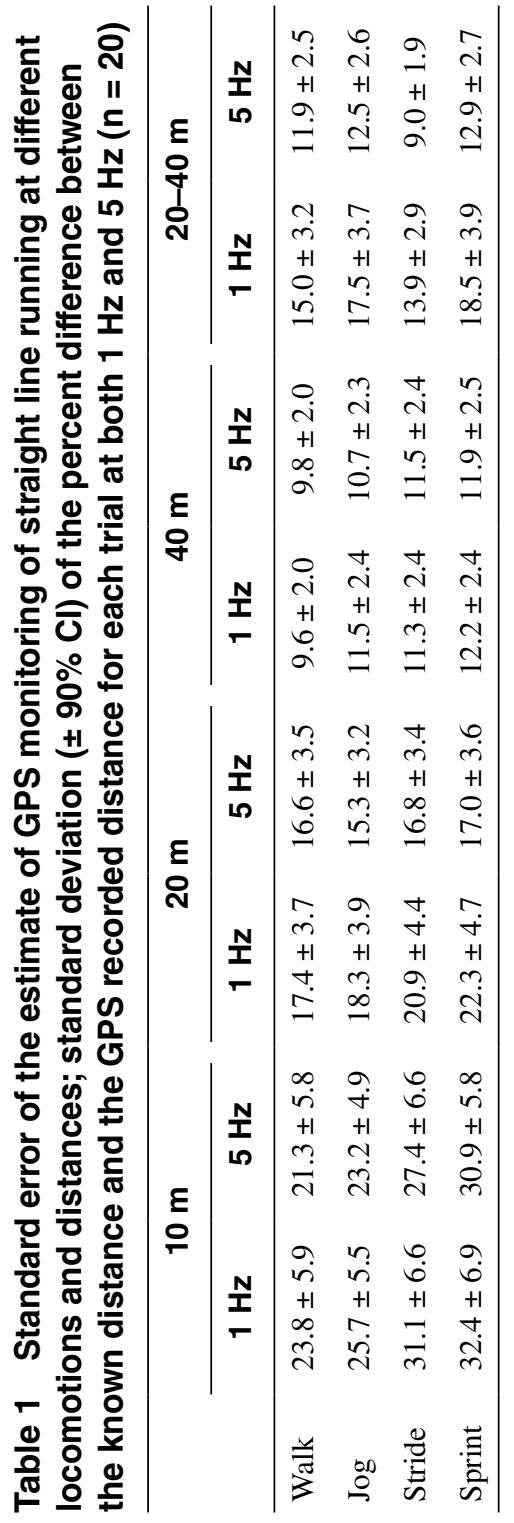


Table 2 Standard error of the estimate of GPS monitoring of two change of direction courses at different locomotion speeds; standard deviation $( \pm 90 \% \mathrm{Cl})$ of the percent difference between the known distance and the GPS recorded distance for each trial at both 1 and 5 $\mathrm{Hz}(\mathrm{n}=\mathbf{2 0})$

\begin{tabular}{lccccc}
\hline & \multicolumn{2}{c}{ Gradual COD } & & \multicolumn{2}{c}{ Tight COD } \\
\cline { 2 - 3 } \cline { 5 - 6 } & $\mathbf{1 ~ H z}$ & $\mathbf{5 ~ H z}$ & & $\mathbf{1 ~ H z}$ & $\mathbf{5 ~ H z}$ \\
\hline Walk & $9.1 \pm 2.4$ & $8.9 \pm 2.3$ & & $12.6 \pm 3.3$ & $9.9 \pm 3.1$ \\
Jog & $10.2 \pm 2.7$ & $9.7 \pm 2.8$ & & $9.0 \pm 2.3$ & $10.6 \pm 2.8$ \\
Stride & $11.5 \pm 3.0$ & $11.0 \pm 3.1$ & & $10.4 \pm 2.7$ & $10.8 \pm 2.8$ \\
Sprint & $12.7 \pm 3.0$ & $11.7 \pm 3.0$ & & $12.5 \pm 3.3$ & $11.5 \pm 3.0$ \\
\hline
\end{tabular}

error from the criterion distance in both the tight and gradual COD regardless of locomotion speed. The GPS underestimated the total distance of the simulated team sport running circuit by $5.7 \pm 0.6 \%$ and $3.7 \pm 0.6 \%$ for 1 and $5 \mathrm{~Hz}$ respectively.

\section{Reliability}

Reliability improved for all locomotor activities moving in a straight line when sampled at the higher rate and over longer distances (Table 5). Regardless of locomotion or distance, GPS was incapable of detecting the SWC for straight line locomotion (TE $>$ SWC, Table 5). The CV for measures over the shorter distances (ie, $10 \mathrm{~m}$ and $20 \mathrm{~m}$ ) were poor $(\mathrm{CV}>10 \%)$ regardless of locomotor activity, speed or sampling rate (Table 5). The higher sample rate and slower movement speed improved all reliability measures regardless of distance (Table 5). The CV measured at $5 \mathrm{~Hz}$ for the 20 to $40 \mathrm{~m}$ split were similar, regardless of locomotion speed with values ranging from $12.1 \%$ (walking) to $9.8 \%$ (sprinting).

Small differences in the reliability measures between the CVs for the tight COD and gradual COD were evident (Table 6). In all cases, the magnitude of the TE was substantially greater than the SWC (Table 6). As locomotion speed in the COD increased, reliability measures marginally improved at higher sampling rates (Table 6). The CV for both the tight and gradual COD tasks were similar to the 40 $\mathrm{m}$ straight line values in jogging, striding and sprinting. However, CV values for COD walking were larger than straight line walking.

The simulated team sport circuit showed good reliability with a $\mathrm{CV}$ of $3.6 \%$ for each sample rate. However, the TE for $1 \mathrm{~Hz}$ : $4.6 \mathrm{~m}(4.1$ to $5.3 \mathrm{~m}, 90 \% \mathrm{CI})$ and $5 \mathrm{~Hz}$ : $4.7 \mathrm{~m}(4.2$ to $5.4 \mathrm{~m}, 90 \% \mathrm{CI})$ was again greater than the $\mathrm{SWC}(1 \mathrm{~Hz}=1.0$ and $5 \mathrm{~Hz}=1.1 \mathrm{~m})$.

\section{Discussion}

The first major finding of this study was that GPS grossly underestimated the criterion distance during sprinting over short distances. This error was evident 


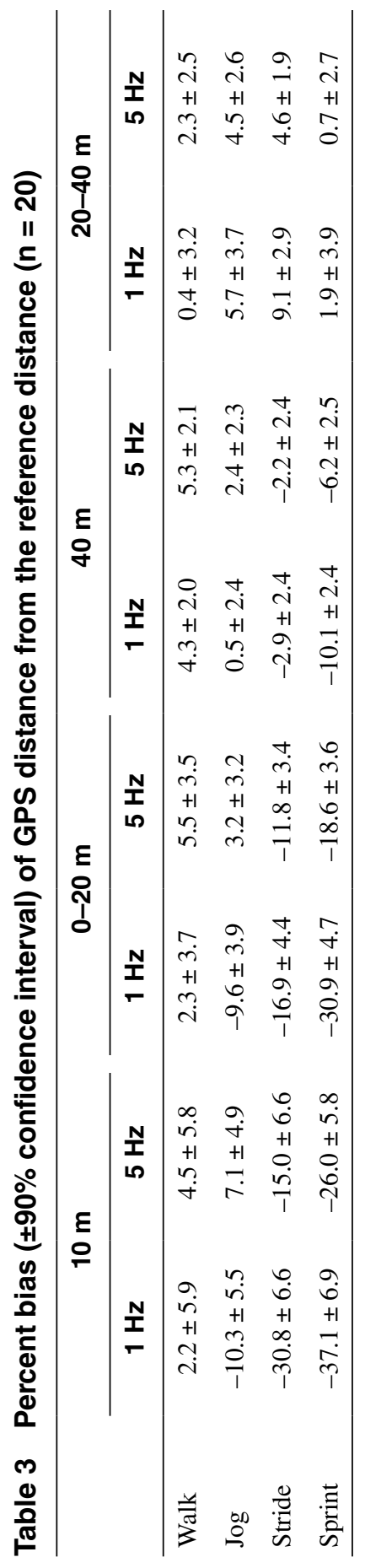


Table 4 Percent bias of GPS monitoring of two change of direction (COD) courses for different locomotion speeds; mean difference of GPS distance from criterion distance expressed as a percentage \pm $90 \%$ confidence intervals $(n=20)$

\begin{tabular}{lccccc}
\hline & \multicolumn{2}{c}{ Gradual COD } & & \multicolumn{2}{c}{ Tight COD } \\
\cline { 2 - 3 } \cline { 5 - 6 } & $\mathbf{1 ~} \mathbf{~ H z}$ & $\mathbf{5 ~ H z}$ & & $\mathbf{1 ~ H z}$ & $\mathbf{5 ~ H z}$ \\
\hline Walk & $-2.0 \pm 2.4$ & $-0.6 \pm 2.3$ & & $-9.2 \pm 3.3$ & $-5.2 \pm 3.1$ \\
Jog & $-6.1 \pm 2.7$ & $-2.3 \pm 2.8$ & & $-13.4 \pm 2.3$ & $-7.1 \pm 2.8$ \\
Stride & $-12.4 \pm 3.0$ & $-7.8 \pm 3.1$ & & $-15.4 \pm 2.7$ & $-10.9 \pm 2.8$ \\
Sprint & $-17.4 \pm 3.0$ & $-12.9 \pm 3.0$ & & $-22.2 \pm 3.3$ & $-15.8 \pm 3.0$ \\
\hline
\end{tabular}

regardless of sampling rate, although higher sampling rate improved validity irrespective of distance and locomotion in the straight line, COD and simulated running circuit trials. The reliability of GPS improved as distance traveled increased but decreased as speed increased. In agreement with previous research ${ }^{6-8,13}$ the GPS devices have acceptable reliability and validity for assessing total distance during longer duration training drills and games. However, the current results show that these devices cannot be used to measure brief, high-intensity sprints, or slow and fast accelerations over distances of less than $20 \mathrm{~m}$, regardless of sampling rate.

The present findings question the efficacy of using GPS technology to quantify high speed movements and accelerations over short distances in team sports. The poor accuracy of these devices for assessing brief accelerations and short sprints may be due to the low number of position measures taken during brief efforts $<3 \mathrm{~s}$. However, changes in velocity also seem to influence the accuracy of the measure. The present results show that the 20 to $40 \mathrm{~m}$ distance measures (ie, postacceleration) are more accurate than the 0 to $20 \mathrm{~m}$ (ie, acceleration) phase of the $40 \mathrm{~m}$ sprint/ stride. This is likely to be associated with the smaller variations in velocity in the final $20 \mathrm{~m}$ of these efforts. Interestingly, there were lower bias values in the 20 to $40 \mathrm{~m}$ phase during sprinting and walking compared with jogging and striding. The higher bias values when jogging and striding might be due to players moving nonconstant velocities during this part of the test. However, it is suggested that future studies need to determine the influence of changes in velocity on measurement error with GPS devices.

The measurement accuracy of GPS decreased during high-intensity COD movements regardless of sample rate. The tight COD demonstrated lower validity than the gradual COD, and this may be due to the increased number of speed changes in the tight COD course. Nonetheless, at slow speeds, the GPS units appear to provide a valid measure of distance for gradual COD activities.

The degree of reliability varied as a function of distance and intensity. In straight line trials, the largest $\mathrm{CV}$ values occurred during striding and sprinting over shorter distances. This is supported by the finding that reliability improved after the acceleration phase of the $40 \mathrm{~m}$ strides and sprints. The poor reliability for these shorter distances may demonstrate the inability of GPS to identify periods of 


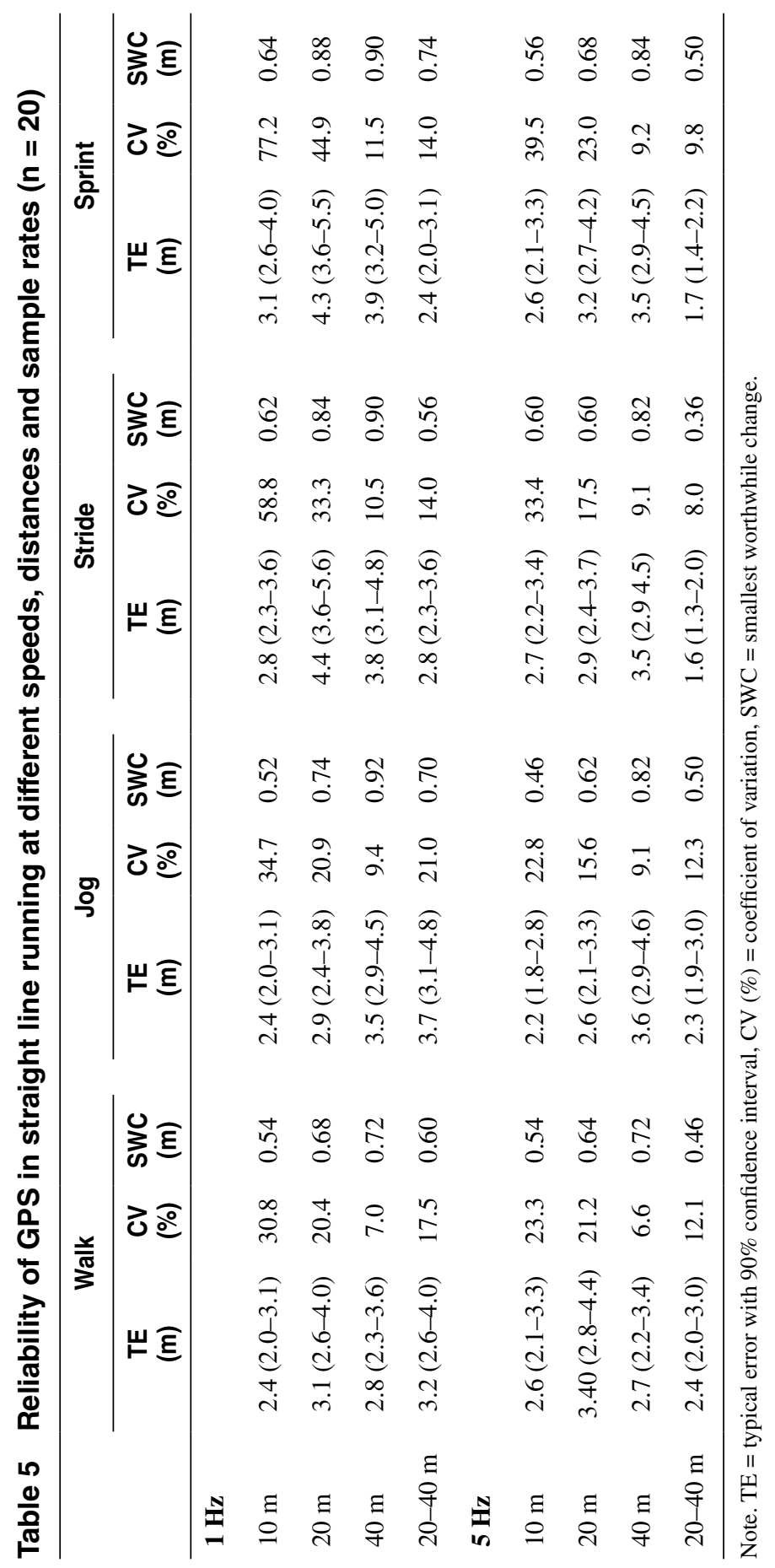




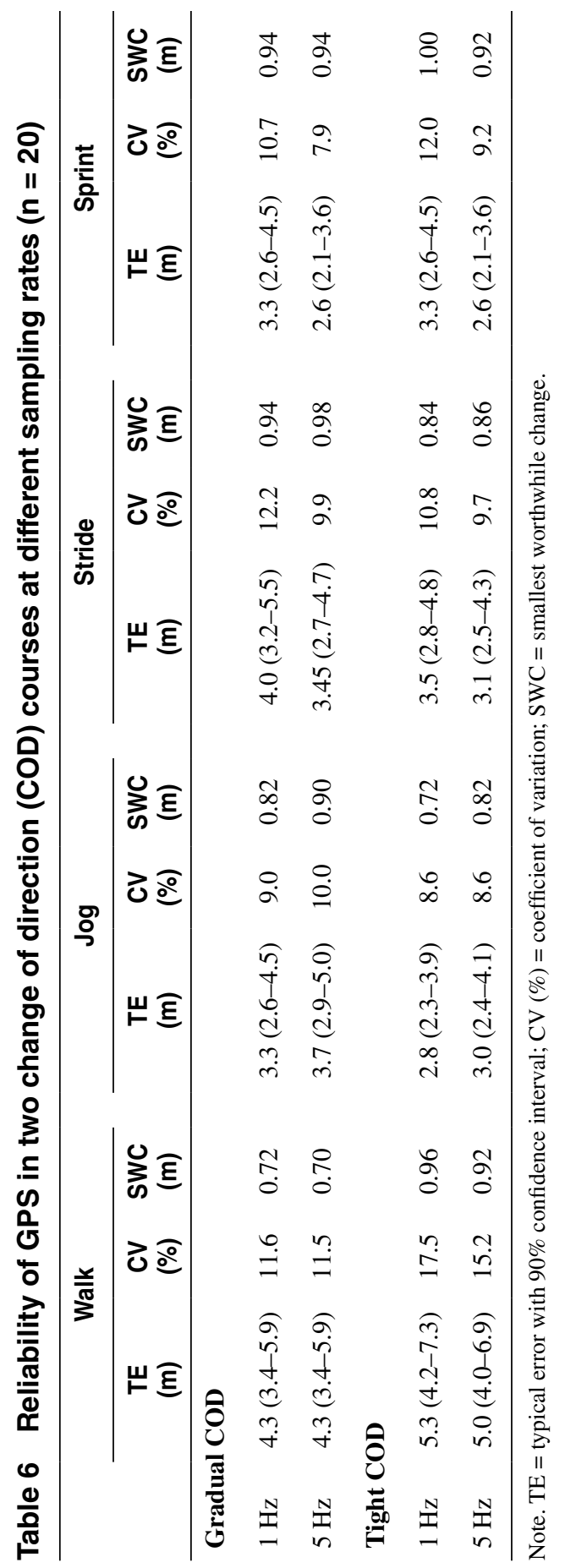


rapid variations in speed. These rapid accelerations over short distances are critical in team sports such as Australian football, soccer, rugby union and hockey, with typical sprint ranges between 10 to $20 \mathrm{~m} \cdot{ }^{14-19}$ Given the larger CV during short, high-intensity efforts, the use of GPS to assess running efforts is inappropriate, especially if data are sampled at $1 \mathrm{~Hz}$.

There was little difference in the reliability of GPS measures between the gradual and tight COD course. Due to the regular changes in direction, acceleration was slower in the agility courses than the straight line course. This most likely explains the improved reliability with the COD courses compared with the straight line movements of similar speeds. Higher sampling frequencies also improved the reliability during high speed measures when sprinting through the COD courses. This suggests that using GPS at a higher sampling rate is more suitable for use in team sports where changes of direction are prevalent. It is possible that some of the error maybe due to differences between the actual and intended pathway. ${ }^{6}$ However, in this study error related to deviations from the intended pathway were minimized with the use of poles placed at each COD and painted lines to ensure minimal movement from the set course.

The simulated team sport circuit is more complex, consisting of a combination of locomotion speeds over straight line and COD movements but over a longer distance. The present results show that the validity and CV were similar to what has been reported for $1 \mathrm{~Hz}$ GPS. ${ }^{6}$ The total distance measured for longer team sport bouts therefore has acceptable accuracy and reliability. Similar to straight line running, longer durations and/or distances, as well as sampling rate improves the measuring precision of these GPS devices.

Collectively, the present results show that the GPS devices used in this study have an acceptable level of validity and reliability for recording movement patterns at lower speeds and for higher sampling rates over longer efforts. The inability of these GPS units to accurately assess movement during rapid variations in speed over short distances, which are critical in team sports, ${ }^{14-19}$ questions the usefulness of these devices for assessing small segments of training or games. In this study, the higher sampling rate improved validity regardless of distance and speed of movement during straight line, COD and simulated team sport running circuit. However, the poor reliability measures, in relation to the SWC, suggest that the GPS provides information that currently is unable to indentify small changes of practical importance. In all cases, magnitude of the typical error was substantially greater than the SWC.

\section{Practical Applications}

GPS microtechnology is currently used in team sports to quantify movement demands in both training and competition. This information can then be used to modify the type, duration and intensity of training, improving the specificity of these sessions. In agreement with previous research, ${ }^{6-8,13}$ the reliability and validity of GPS to estimate longer distances appears to be acceptable (ie, <10\%) for game and training purposes. However, currently available GPS systems maybe limited for the assessment of brief, high speed straight line running, accelerations or efforts involving a change of direction. These results also show that an increased sample rate improves the reliability and validity of GPS devices. Further development of 
GPS technology is required before practitioners can be confident about the suitability of GPS for the quantification of short, high intensity intermittent running movement patterns common in team sports. In particular, care needs to be taken when interpreting data on single sprints or small changes in direction and velocity. The degree of error may reduce the ability to report distance covered in narrow speed zones. At present, practitioners may be limited to using variables such as total distance obtained for full matches or periods of play.

\section{References}

1. Roberts S, Trewartha G, Stokes K. A comparison of time-motion analysis methods for field-based sports. Int J Sports Physiol Perform. 2006;1:388-399.

2. Carling C, Bloomfield J, Nelsen L, Reilly T. The role of motion analysis in elite soccer: contemporary performance measurement techniques and work rate data. Sports Med. 2008;38:839-862.

3. Dobson B, Keogh JWL. Methodological issues for the application of time-motion analysis research. Strength Condit J. 2007;29:48-55.

4. Aughey RJ, Falloon C. Real-time versus post-game GPS data in team sports. J Sci Med Sport. 2009;13:348-349.

5. Barbero-Álvarez JC, Coutts A, Granda J, Barbero-Álvarez V, Castagna C. The validity and reliability of a global positioning satellite system device to assess speed and repeated sprint ability (RSA) in athletes. $J$ Sci Med Sport. 2010;13:232-235.

6. Coutts AJ, Duffield R. Validity and reliability of GPS units for measuring movement demands of team sports. J Sci Med Sport. 2010;13:133-135.

7. Edgecomb SJ, Norton KI. Comparison of global positioning and computer-based tracking systems for measuring player movement distance during Australian football. J Sci Med Sport. 2006;9:25-32.

8. MacLeod H, Morris J, Nevill A, Sunderland C. The validity of a non-differential global positioning system for assessing player movement patterns in field hockey. $J$ Sports Sci. 2009;27:121-128.

9. Rampinini E, Coutts AJ, Castagna C, Sassi R, Impellizzeri FM. Variation in top-level soccer performance. Int J Sports Med. 2007;28:1018-1024.

10. Rampinini E, Impellizzeri FM, Castagna C, Coutts AJ, Wisløff U. Technical performance during soccer matches of the Italian Serie A league: Effect of fatigue and competitive level. J Sci Med Sport. 2009;12:227-233.

11. Bradley PS, Sheldon W, Wooster B, Olsen P, Boanas P, Krustrup P. High-intensity running in FA Premier League soccer matches. J Sports Sci. 2009;27:159-168.

12. Coutts AJ, Quinn J, Hocking J, Castagna C, Rampinini E. Match running performance in elite Australian Rules Football. J Sci Med Sport. 2010;13:543-548.

13. Petersen C, Pyne D, Portus M, Dawson B. Validity and reliability of GPS units to monitor cricket-specific movement patterns. Int J Sports Physiol Perform. 2009;4:381-393.

14. Duthie GM, Pyne DB, Marsh DJ, Hooper SL. Sprint patterns in rugby union players during competition. J Strength Cond Res. 2006;20:208-214.

15. Sirotic AC, Coutts AJ, Knowles H, Catterick C. A comparison of match demands between elite and semi-elite rugby league competition. J Sports Sci. 2009;27:203-211.

16. Rampinini E, Bishop D, Marcora SM, Ferrari Bravo D, Sassi R, Impellizzeri FM. Validity of simple field tests as indicators of match-related physical performance in top-level professional soccer players. Int J Sports Med. 2007;28:228-237.

17. Impellizzeri FM, Rampinini E, Castagna C, et al. Validity of a repeated-sprint test for football. Int J Sports Med. 2008;29(11):899-905 
18. Spencer M, Lawrence S, Rechichi C, Bishop D, Dawson B, Goodman C. Time-motion analysis of elite field hockey, with special reference to repeated-sprint activity. J Sports Sci. 2004;22:843-850.

19. Dawson B, Hopkinson R, Appleby B, Stewart G, Roberts C. Player movement patterns and game activities in the Australian Football League. J Sci Med Sport. 2004;7:278-291.

20. Bloomfield J, Polman R, O'Donoghue P. Physical demands of different positions in FA Premier League soccer. J Sports Sci Med. 2007;6:63-70.

21. Mujika I, Santisteban J, Impellizzeri FM, Castagna C. Fitness determinants of success in men's and women's football. J Sports Sci. 2009;27:107-114.

22. Witte T, Wilson A. Accuracy of non-differential GPS for the determination of speed over ground. J Biomech. 2004;37:1891-1898.

23. Witte T, Wilson A. Accuracy of WAAS-enabled GPS for the determination of position and speed over ground. J Biomech. 2005;38:1717-1722.

24. Bishop D, Spencer M, Duffield R, Lawrence $S$. The validity of a repeated sprint ability test. J Sci Med Sport. 2001;4:19-29.

25. Pyne D. Interpreting the results of fitness testing. Paper presented at: International Science and Football Symposium, 2003; Melbourne, Australia.

26. Duthie G, Pyne D, Hooper S. The reliability of video based time motion analysis. $J$ Hum Mov Stud. 2003;44:259-272. 